\title{
Vitamin D supplements: Magic pill or overkill?
}

\author{
Ronald B. Brown ${ }^{1}$, Fatme Al Anouti ${ }^{2}$, Mohammed S. Razzaque ${ }^{3}$
}

\begin{abstract}
${ }^{1}$ Post-graduate student, School of Public Health and Health Systems, University of Waterloo, Waterloo, Ontario, Canada. ${ }^{2}$ Associate Professor, College of Sustainability Sciences and Humanities, Zayed University, Abu Dhabi, UAE. ${ }^{3}$ Professor of Pathology, Department of Pathology, Saba University School of Medicine, Devens, Massachusetts, USA; Department of Applied Oral Sciences, Forsyth Institute, Harvard School of Dental Medicine Affiliate, Cambridge, Massachusetts, USA.
\end{abstract}

Vitamin D is a multifunctional micronutrient that exerts hormonal effects on many organs of the body. 1,25 dihydroxyvitamin $\mathrm{D}$ is the active metabolite of vitamin $\mathrm{D}$, formed by dual hydroxylation in the liver and kidney (Figure 1). ${ }^{1}$ Circulating 1,25 dihydroxyvitamin D exerts biological functions, mainly through interaction with vitamin $\mathrm{D}$ receptors (VDRs), and can increase the intestinal absorption of mineral ions such as calcium and phosphate; it can also suppress the biosynthesis of parathyroid hormone. High serum levels of 1,25 dihydroxyvitamin $\mathrm{D}$ are believed to increase osteoclastic bone resorption, which mobilizes calcium and phosphate from bone. Of relevance, vitamin D is a strong inducer of fibroblast growth factor 23 (FGF23) and klotho, two important homeostatic regulators of calcium and phosphate metabolism. ${ }^{2-4}$

An association between hypovitaminosis D and ageassociated disorders, including osteoporosis, cancer, diabetes, autoimmune disorders, hypertension, atherosclerosis, and muscle weakness is reported; a similar association is also noted with neurological disorders

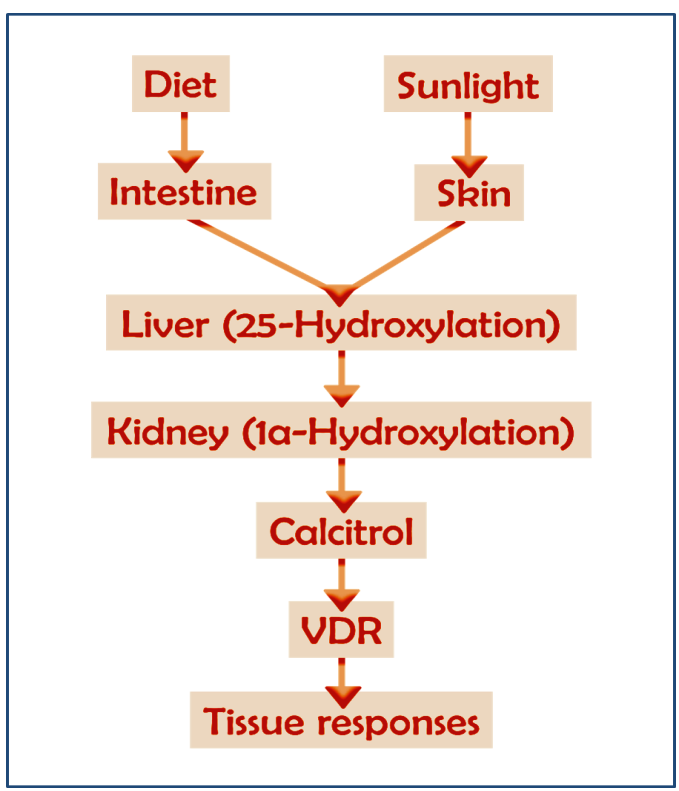

Fig 1: Different stages of vitamin D synthesis ${ }^{1}$ Simplified diagram of the different stages of vitamin D synthesis. Please note that the molecular regulation of vitamin D synthesis is much complex, and for the simplicity, we have only mentioned some of the essential events of vitamin synthesis. ${ }^{1}$ VDR: vitamin D receptor. including multiple sclerosis, Alzheimer's disease, Parkinson's disease, and schizophrenia. ${ }^{5}$ However, it is not yet clear whether such associations are a cause or consequence of vitamin $\mathrm{D}$ deficiency. In addition, calcium supplements along with vitamin $\mathrm{D}$ are commonly used to delay the progression of osteoporosis, but the expected benefit of calcium and vitamin $\mathrm{D}$ supplements are minimized by the potential risk of cardiovascular events, including vascular calcification. ${ }^{6,8}$ In fact, studies have documented that calcium supplements increased the risk of myocardial infarction and stroke, as high as $30 \%{ }^{9,10}$ Consumption of vitamin D and calcium supplements can also enhance the risk of developing cardiovascular calcification in the elderly. ${ }^{11-13}$ We believe that harmful consequences of cardiovascular effects following calcium and vitamin D ingestion may be partly related to dysregulated phosphate balance. ${ }^{1,14-16}$

A wide range of vitamin-D fortified food is currently consumed with the anticipation that such fortified food and drink can maintain bone health by preventing skeletal disorders, including rickets. Food fortification is an accepted practice in many countries, although many types of food fortification were historically outlawed in several European countries. ${ }^{17}$ This policy was strengthened in Europe after England's epidemic of adverse effects in children who were fed vitamin-D fortified foods during the 1950s. As well as promoting sales of fortified "junk" food, investigators cited potential fortification-related risks from ingesting excessive micronutrients that do not exist in natural whole foods, which may lead to metabolic imbalances.

Nutrition authorities also point out that supplements cannot replace food as a primary source of nutrients in a balanced diet. Nevertheless, the 1994 Dietary Supplement Health and Education Act passed by the U.S. Congress allows supplements to be released on the market without evidence of safety or effectiveness, thereby fueling an explosive growth in marketing suspect dietary supplements. ${ }^{18}$ As a result, rather than use as a backup nutritional plan, there may be an increasing reliance on supplementation and fortification to balance an unhealthy diet, which undermines the public's motivation to obtain essential nutrients from consuming natural whole foods. The false sense of security and convenience provided by swallowing a pill

Correspondence: Dr. Mohammed S. Razzaque, Professor of Pathology, Saba University School of Medicine, Church Street, Saba, Caribisch Nederland; Devens, MA 01434, USA. E-mail: mrazzaque@,forsyth.org, m.razzaque@saba.edu.

South East Asia Journal of Public Health 2015;5(2):1-3 (C) 2015 Brown et al., publisher and licensee Public Health Foundation Bangladesh. This is an Open Access article which permits unrestricted non-commercial use, provided the original work is properly cited. DOI: http://dx.doi.org/10.3329/seajph.v5i2.28301 
or eating fortified 'junk food' implies that supplementation and fortification may reinforce dependency on supplements as a quick fix rather than encourage healthier eating practices. For example, most people who rely on supplements instead of food said they "find it hard to eat a balanced diet". ${ }^{19}$

Furthermore, serum 25-hydroxyvitamin D levels in many countries are generally lower than in Western populations where vitamin D supplementation and food fortification is more common. Thus, by Western standards, vitamin D deficiency in global populations is apparent, but is it a real phenomenon? Studies of 25 hydroxyvitamin within global populations need to document whether applying the higher Western standard of serum concentrations is a valid indicator of true vitamin D deficiency. For example, global populations with darker skin color, greater skin coverage from clothing, and less exposure to natural sunlight have little trouble maintaining health with lower serum 25-hydroxyvitamin D levels. Promoting higher 25-hydroxyvitamin D levels may benefit the supplement industry and the practitioners who prescribe supplements, but it is not supported by evidence of health outcomes in global populations. In fact, bone health in global populations with lower vitamin D status, such as in Asian countries, is often far better than in vitamin-D supplemented populations in North America and Europe. ${ }^{20}$

It has been known for almost a century that moderate sun exposure is sufficient to ensure adequate vitamin D levels and eliminate rickets. ${ }^{21}$ It is believed that that "Ten to 15 minutes of sunshine three times weekly is enough to produce the body's requirement of vitamin D. The sun needs to shine on the skin of your face, arms, back, or legs (without sunscreen)" This amount of vitamin $\mathrm{D}$ is all that is required to promote healthy bone growth and prevent rickets; unfortunately, rickets is once again reappearing as safe sun exposure falls out of favor with the general public over concerns of skin cancer. $^{22}$ Ironically, evidence shows that outdoor workers have lower skin cancer risk than indoor workers.

In summary, vitamin $\mathrm{D}$ produced naturally from adequate exposure to sunlight is necessary to prevent health problems like rickets, which is unquestioned. That vitamin D supplementation is necessary for health remains questionable, and uncontrolled use of vitamin D supplements, in addition to consumption of vitamin D fortified food, may induce unexpected adverse effects. The overall benefits of calcium and vitamin D supplementation to osteoporotic patients is not yet settled. Of relevance, citing the U.S. Preventive Services Task Force investigation of vitamin D and calcium supplementation, Nestle et al. ${ }^{23}$ pointed out that there is no solid evidence of benefits from vitamin D supplementation that are worth even the smallest risk. Additionally, experimental studies suggest that cardiovascular calcification due to excessive levels of calcium and vitamin $\mathrm{D}$ may be partly related to dysregulated phosphate balance. ${ }^{14,24-29}$
Several final points remain to be raised regarding vitamin supplements. The argument that these substances are safe for use until toxic effects appear is as incongruous as arguing that tobacco use is safe until cancer appears. Safety is not exclusively a quantitative issue concerning the accumulation of toxic effects, but also includes the qualitative issue concerning the nature of the substance's interactions with the body. Many supplements derived from animal and plant extracts as well as laboratory synthesized analogues do not function as natural or native nutrients. They are qualitatively foreign substances to the human body, they often produce a pharmacological effect by interfering with normal physiological mechanisms, and they also have adverse effects as do most of the drugs. It is not up to independent researchers to verify that these substances are unsafe and ineffective for use; the onus should rightfully be on the manufacturers and promotors of these substances to prove that their supplements and patented pharmaceutical products are safe and effective, especially for long-term use, but the evidence shows they have neglected to do so by effectively using the loopholes of regulations.

\section{References}

1. Razzaque MS. The dualistic role of vitamin D in vascular calcifications. Kidney Int 2011;79:70814.

2. Razzaque MS. Bone-kidney axis in systemic phosphate turnover. Arch Biochem Biophys 2014;561:154-8.

3. Razzaque MS. The FGF23-Klotho axis: endocrine regulation of phosphate homeostasis. Nat Rev Endocrinol 2009;5:611-9.

4. Razzaque MS, Lanske B. The emerging role of the fibroblast growth factor-23-klotho axis in renal regulation of phosphate homeostasis. $J$ Endocrinol 2007;194:1-10.

5. Annweiler C, Schott AM, Berrut G, Chauvire V, Le Gall D, Inzitari M, et al. Vitamin D and ageing: neurological issues. Neuropsychobiology 2010;62:139-50

6. Reid IR, Bolland MJ. Calcium supplementation and vascular disease. Climacteric. 2008;11:2806.

7. Reid IR, Bolland MJ, Grey A. Effect of calcium supplementation on hip fractures. Osteoporos Int 2008;19:1119-23.

8. Reid IR, Bolland MJ, Avenell A, Grey A. Cardiovascular effects of calcium supplementation. Osteoporos Int 2011;22:164958.

9. Bolland MJ, Barber PA, Doughty RN, Mason B, Horne A, Ames R, et al., Vascular events in healthy older women receiving calcium supplementation: randomised controlled trial. BMJ 2008;336:262-66.

10. Bolland MJ, Grey A, Avenell A, Gamble GD, 
Reid IR. Calcium supplements with or without vitamin $\mathrm{D}$ and risk of cardiovascular events: reanalysis of the Women's Health Initiative limited access dataset and meta-analysis. $B M J$ 2011;342:d2040.

11. Trived DP, Doll R, Khaw KT. Effect of four monthly oral vitamin D3 (cholecalciferol) supplementation on fractures and mortality in men and women living in the community: randomised double blind controlled trial. $B M J$ 2003;326:469.

12. Heikinheimo RJ, Inkovaara JA, Harju EJ, Haavisto MV, Kaarela RH, Kataja JM, Kokko AM, Kolho LA, Rajala SA. Annual injection of vitamin D and fractures of aged bones. Calcif Tissue Int 1992;51:105-10.

13. Jackson RD, LaCroix AZ, Gass M, Wallace RB, Robbins J, Lewis CE, eCalcium plus vitamin D supplementation and the risk of fractures. NEJM 2006;354:669-83.

14. Razzaque MS. Phosphate toxicity: new insights into an old problem. Clin Sci (Lond) 2011;120:91 -7 .

15. Razzaque MS. Osteo-renal regulation of systemic phosphate metabolism. IUBMB Life 2011;63:240-7.

16. Razzaque MS. FGF23-mediated regulation of systemic phosphate homeostasis: is Klotho an essential player? Am J Physiol Renal Physiol 2009;296:F470-6.

17. Bonner G, Warwick H, Barnardo $\mathrm{M}$, Commission F. Fortification Examined: How Added Nutrients Can Undermine Good Nutrition; a Survey of 260 Food Products with Added Vitamins and Minerals. London: Food Commission (UK) Ltd, 1999.

18. Redberg RF. Vitamin supplements: more cost than value. Arch Intern Med 2011;171:1634-5.
19. Neuhouser ML, Patterson RE, Levy L. Motivations for Using Vitamin and Mineral Supplements. J Am Dietetic Assoc 1999;99:8514.

20. Dhanwal DK, Dennison EM, Harvey NC, Cooper C. Epidemiology of hip fracture: Worldwide geographic variation. Indian $J$ Orthop 2011;45:15-22.

21. Hess AF, Unger LJ. The cure of infantile rickets by sunlight: Preliminary note. JAMA 1921;77:39.

22. Holick MF. Resurrection of vitamin D deficiency and rickets. J Clin Invest 2006;116:2062-72.

23. Nestle M, Nesheim MC. To supplement or not to supplement: the U.S. Preventive Services Task Force recommendations on calcium and vitamin D. Ann Intern Med 2013;158:701-2.

24. Brown RB, Razzaque MS. Dysregulation of phosphate metabolism and conditions associated with phosphate toxicity. Bonekey Rep 2015;4:705.

25. Brown RB, Razzaque MS. Phosphate toxicity: a stealth biochemical stress factor? Med Mol Morphol 2016;49:1-4.

26. Brown RB, Haq A, Stanford CF, Razzaque MS. Vitamin D, phosphate, and vasculotoxicity. Can J Physiol Pharmacol 2015;93:1077-82.

27. Razzaque MS. Phosphate toxicity and vascular mineralization. Contrib Nephrol 2013;180:74-85.

28. Ohnishi M, Razzaque MS. Dietary and genetic evidence for phosphate toxicity accelerating mammalian aging. FASEB J 2010;24:3562-71.

29. Ohnishi M, Nakatani T, Lanske B, Razzaque MS. In vivo genetic evidence for suppressing vascular and soft-tissue calcification through the reduction of serum phosphate levels, even in the presence of high serum calcium and 1,25dihydroxyvitamin D levels. Circ Cardiovasc Genet 2009;2:583-90. 